\title{
Chaotic and Mixed Mode Oscillation Scenarios in Semiconductor Devices: Generation and Synchronization
}

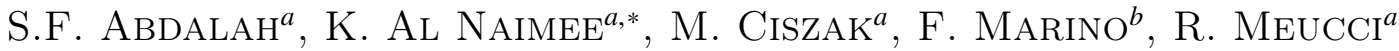 \\ AND F.T. ARECCHI ${ }^{a, b}$ \\ ${ }^{a}$ Istituto Nazionale di Ottica, Largo E. Fermi 6, 50125, Florence, Italy \\ ${ }^{b}$ Dipartimento di Fisica, Università di Firenze, INFN, Sezione di Firenze \\ via Sansone 1, I-50019 Sesto Fiorentino (FI), Italy
}

\begin{abstract}
We demonstrate the existence of chaotic spiking and mixed mode oscillations sequences in the dynamics of semiconductor devices (laser diode and light emitting diode) with ac-coupled optoelectronic feedback. We eventually show that this regime is the result of an incomplete homoclinic scenario to a saddle-focus, where an exact homoclinic connection does not occur. The synchronization scenarios have been shown.
\end{abstract}

PACS: 42.60.Mi

\section{Introduction}

Irregular spiking sequences in biological, chemical and electronic systems have been frequently observed to be the result of multiple timescale dynamics [1]. Indeed, a variety of natural systems showing this behaviour (neural cells and cardiac tissues, chemical reactions [2], to name just a few) can be mathematically described by means of slow and fast variables coupled together. In two-dimensional phase spaces, irregular spiking can forcibly appear only in the presence of noise close to fixed points limit cycles bifurcations (the Andronov saddle-node collisions, sub and super critical Hopf bifurcations, etc.). In contrast, higher dimensional systems can support more varied and complex dynamics, such as mixed-mode oscillations (MMO) and erratic bursting [2,3].

In many cases [4-7] these phenomena can be understood in terms of a paradigmatic model known as the Shilnikov homoclinic chaos (HC) [8]. HC may arise in three-dimensional phase space when a growing periodic orbit approaches a saddle-focus becoming homoclinic to it, i.e. biasymptotic for $t \rightarrow \pm 1$. Typical time series consists of large pulses (associated with a homoclinic orbit in the phase space) separated by irregular time intervals in which the system displays small-amplitude chaotic oscillations. This behavior arises in agreement with the Shilnikov theorem, which predicts the occurrence of complex dynamics near homoclinicity whenever the saddle-focus, with linearized eigenvalues $(\mu, \delta \pm i \omega),(\delta, \mu>0)$, satisfies the condition $|\delta / \mu|<1$.

* corresponding author; e-mail: Kais.al-naimee@ino.it
In the following we report the occurrence of chaotic and MMO spiking in semiconductor devices (laser diodes (LD) and LED) with ac-coupled nonlinear optoelectronic feedback. The solitary laser dynamics is ruled by two coupled variables (intensity and population inversion) evolving with two very different characteristic timescales. The introduction of a third degree of freedom (and a third timescale) describing the ac-feedback loop, leads to a three-dimensional slow-fast system displaying a transition from a stable steady state to periodic spiking sequences as the dc-pumping current is varied.

\section{Chaos generation in semiconductor devices}

We consider a closed-loop optical system, consisting of semiconductor devices (LD or LED) with ac-coupled nonlinear optoelectronic feedback. The output light is sent to a photodetector producing a current proportional to the optical intensity. The corresponding signal is sent to a variable gain amplifier characterized by a nonlinear transfer function of the form $f(w)=A w /(1+s w)$, where $A$ is the amplifier gain and $s$ a saturation coefficient, and then fed back to the injection current of the laser. The feedback strength is determined by the amplifier gain, while its high-pass frequency cut-off can be varied (between $1 \mathrm{~Hz}$ and $100 \mathrm{kHz}$ ) by means of a tunable high-pass filter. In the upper part of Fig. 1a, corresponding to the lowest current, the detected optical power is stable. As the current is delicately increased a transition to a chaotically spiking regime is observed (Fig. 1b). This scenario is qualitatively similar to homoclinic chaos (Fig. 1d). Further increase of the current makes the firing rate higher until a periodic regime is eventually reached (Fig. 1c) [9]. 

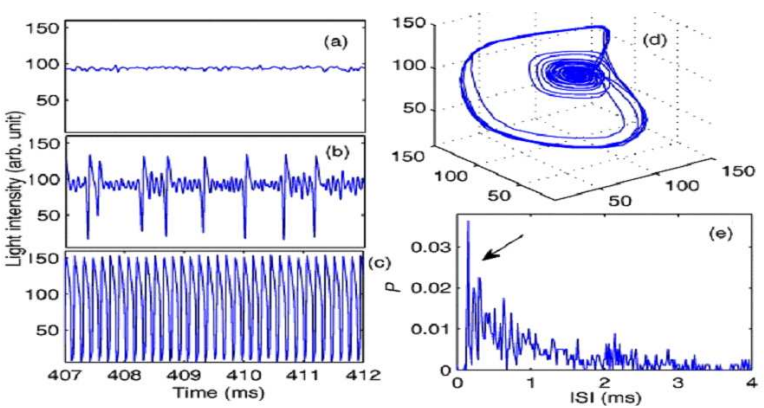

Fig. 1. Transition from a stationary steady state to chaotic spiking and eventually periodic self-oscillations as the dc-pumping current is varied. (a) $8.700 \mathrm{~mA}$, (b) $8.763 \mathrm{~mA}$ and (c) $9.050 \mathrm{~mA}$. (d) Experimental reconstruction of the phase portrait. (e) The corresponding experimental inter spike interval (ISI) probability distribution for the chaotic spiking regime.

The chaotic spiking regime can be understood in terms of excitability of a chaotic attractor, where the small chaotic background spontaneously triggers excitable spikes in an erratic but deterministic sequence. The time scale of these dynamics, much slower respect to typical carriers time scales $(\approx 1 \mathrm{~ns})$, is fully determined by the high-pass filter in the feedback loop [9].

Although similar to the well known Shilnikov homoclinic chaos observed in various physical systems [10-12] here the attractor structure is substantially different since an exact homoclinic connection to a saddle-focus does not occur. Further increase of the current makes the firing rate higher until a periodic regime is eventually reached (right part in Fig. 2). As the large pulses, these oscillations can be decomposed into a sequence of periods of slow motion, near extreme, separated by faster relaxations between them. This behavior (relaxation oscillations) is typical in multiple time-scales nonlinear systems. The same dynamical sequence can be obtained as the pumping current is kept constant and the amplifier gain is changed.

In addition of the laser diode, incomplete homoclinic chaotic and MMO behaviour can be seen by the LED utilizing the same configuration above (Fig. 2).

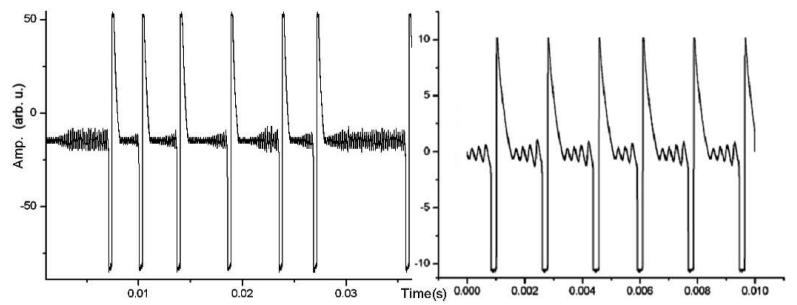

Fig. 2. Chaotical spiking (a) and the MMO (b) regimes of LED.

\section{Synchronization}

Experimental results provided for 2 coupled systems, revealed that the transition to synchronization is not continuous as the control parameter, in our case the bias strength $B$, is varying. To describe quantitatively these abrupt changes we characterize the degree of order in the system by means of entropy $S$ (see Ref. [12]). It is calculated from the distribution of the response times $t_{\mathrm{r}}$ in the time series. When the coupling is zero, this distribution is such, that the information on a site gives no information on the other ones. Increasing the coupling, we observe the birth of peaks for fixed time differences, due to the time correlation between spikes at adjacent sites. The entropy $S$ is defined as follows:

$$
S=\sum p\left(t_{\mathrm{r}}\right) \ln p\left(t_{\mathrm{r}}\right)
$$

where $p\left(t_{\mathrm{r}}\right)$ is the probability distribution of $t_{\mathrm{r}}$. The time series for two LEDs coupled with bias bidirectionally are shown in Fig. 3. The coupling scheme for several oscillators $\left(x_{i}\right)$ can be represented by the simple relation

$$
\dot{x}_{i}=f\left(x_{i}\right)+K_{1} \sum_{j<i}\left(x_{j}-x_{i}\right)+K_{2} \sum_{j>i}\left(x_{j}-x_{i}+B_{i}\right),
$$

where $B$ is the bias current, meanwhile $K_{1}$ and $K_{2}$ are the fixed-valued coupling matrices.

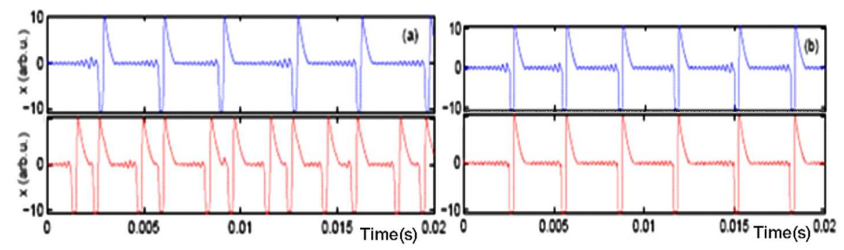

Fig. 3. Time series for two bidirectional coupled LEDs in (a) desynchronized and (b) synchronized state.

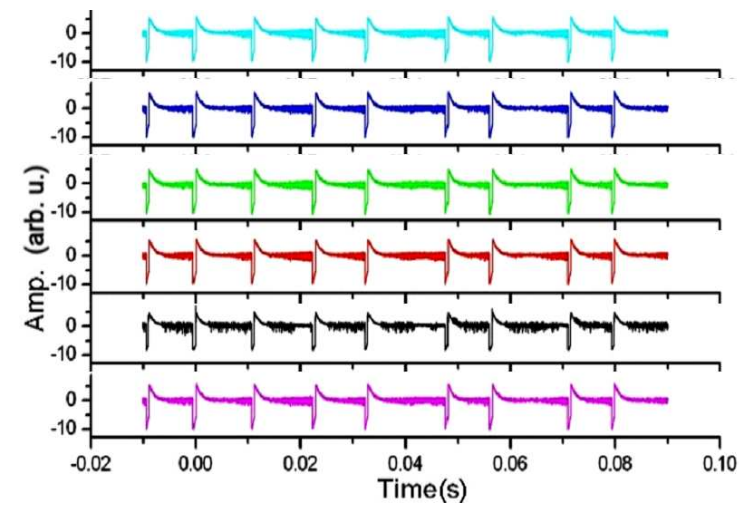

Fig. 5. Synchronized six LED oscillators in spiking regime. 

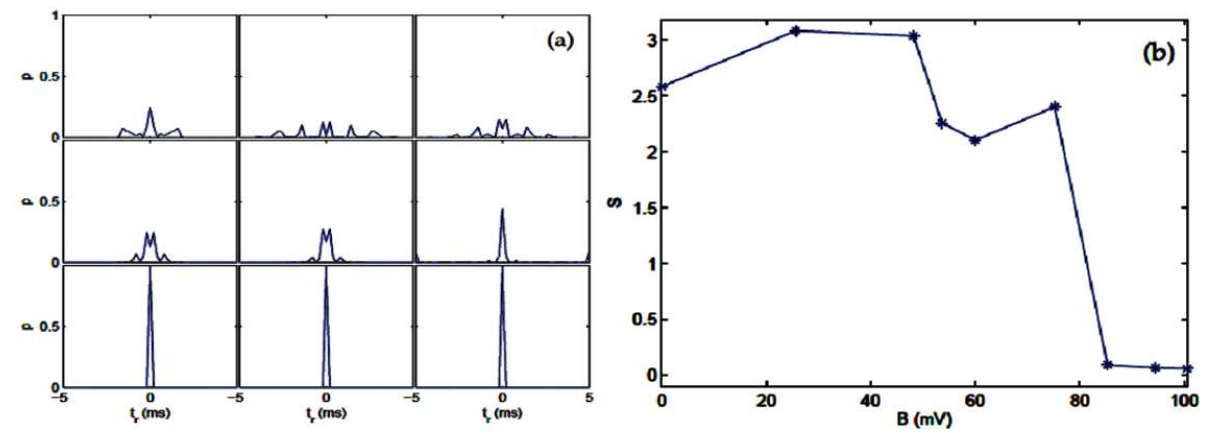

Fig. 4. (a) Distribution of the response times, (b) corresponding entropy versus bias $B$ strength for two bidirectionnally coupled LEDs.

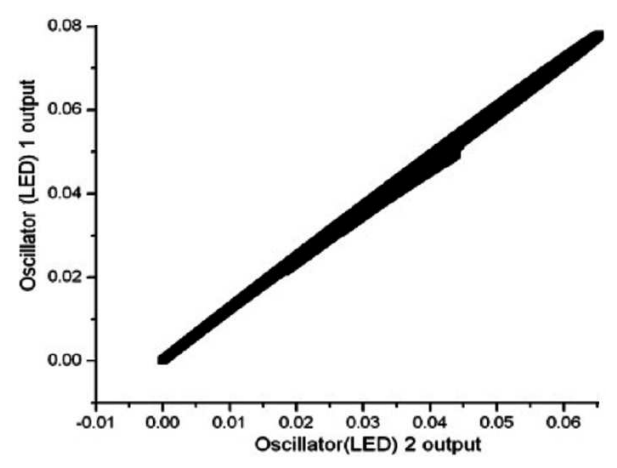

Fig. 6. The correlation between two semiconductor devices (LEDs) in chaotic regime: numerical results.

Let us note that in the case of $N=2$ systems, the implementation of Eq. (2) requires only one control bias $B$. The corresponding probability distributions are plotted in Fig. 4a. It can be seen that as the control bias is changing, the probability distribution becomes sharper giving rise to a low entropy values as reported in Fig. 4b.

Six LEDs have been coupled, in this case the feedback strength and the bias used to feed all of the oscillators. The modulation of the control bias enabled to reach synchronization, Fig. 5.

Numerically, the bifurcation diagram is computed from our system over a small interval contiguous to the initial Hopf bifurcation. Two LED oscillators (in chaotic or MMOs regime) have been synchronized bidirectional by means of differential amplifier. The coupling strength plays a crucial rule in synchronization process. The correlation between two LED outputs is shown in Fig. 6 .

In conclusion, we demonstrate experimentally the existence of slow chaotic spiking sequences in the dynamics of semiconductor devices (LD and LED) with ac-coupled optoelectronic feedback. Experiments concerning syn- chronization phenomena in laser arrays are currently in preparation and will be the subject of future work.

\section{Acknowledgments}

S.F.A. acknowledges the ICTP-TRIL program for financial support.

\section{References}

[1] C.K. Jones, A.I. Khibnik, Multiple Time-Scale Dynamic Systems, IMA Proc., Vol. 122, Springer, New York 2000.

[2] E. Izhikevich, Int. J. Bifurcation Chaos 10, 1171 (2000).

[3] F.N. Albahadily, J. Ringland, M. Schell, J. Chem. Phys. 90, 813 (1989).

[4] C. Vidal, in: Chaos and Order in Nature, Ed. H. Haken, Springer, Berlin 1981, p. 69.

[5] J. Elezgaray, A. Arneodo, Phys. Rev. Lett. 68, 714 (1992).

[6] T. Braun, J.A. Lisboa, J. Gallas, Phys. Rev. Lett. 68, 2770 (1992).

[7] P. Saparin, M.A. Zaks, J. Kurths, A. Voss, V.S. Anishchenko, Phys. Rev. Lett. 54, 737 (1996); J.J. Zebrowski, R. Baranowski, Phys. Rev. E 67, 056216 (2003).

[8] M.T.M. Kope, P. Gaspard, J.H. Sluyters, J. Chem. Phys. 97, 8250 (1992).

[9] K. Al-Naimee, F. Marino, M. Ciszak, R. Meucci, F.T. Arecchi, New J. Phys. 11, 073022 (2009).

[10] F. Marino, F. Marin, S. Balle, O. Piro, Phys. Rev. Lett. 98, 074104 (2007).

[11] V. Petrov, S. Scott, K. Showalter, J. Chem. Phys. 97, 6191 (1992).

[12] M. Ciszak, A. Montina, F.T. Arecchi, Phys. Rev. E 78, $016202(2008)$ 